\title{
EL CAMINO GADAMERIANO HACIA LA HERMENÉUTICA ONTOLÓGICA
}

\author{
Jesús CONILL \\ Universidad de Valencia
}

RESUMEN. Tras considerar la situación vital desde la que se produce el encuentro de Gadamer con la filosofía en el ámbiro neokantiano de las Escuelas de Breslau y Marburgo, se expone el impacto que le produjo Heidegger durante el semestre de verano de 1923 en Friburgo a raíz del curso sobre "Hermenéutica de la facticidad" como base para una nueva ontología. Desde esta plataforma filosófica, enriquecida con sus estudios de filología clásica, Gadamer lleva adelante su propio proyecto de hermeneutización ontológica histórica frente al planteamiento epistemológico. Aquí se destacan no sólo las contribuciones de la analítica hermenéutica gadameriana de la comprensión en el debate filosófico contemporáneo, sino también las insuficiencias de su análisis de la experiencia. Y, por último, se propone una vía de mejor resolución contando con las aportaciones de la filosofía española a una nueva analítica de la "razón experiencial» en sus diversos registros (Unamuno, Machado, Ortega, Zubiri, Zambrano, Laín, Marías).

\section{La situación vital}

Nació Hans-Georg Gadamer en Breslau en el año -para la filosofía contemporánea- emblemático de 1900, en el que murió Nietzsche, Husserl publicó el primer volumen de las Investigaciones Lógicas, Dilthey dio a conocer su trabajo sobre el origen de la hermenéutica ("Die Entstehung der Hermeneutik») y Freud irrumpió con La interpretación de los sueños.

Quienes han estudiado la biografía de Gadamer y los recuerdos de su propia autobiografía destacan que su vida estuvo marcada por una educación autorita- 
ria — prusiana - por parte de su padre, ya que quedó huérfano de madre muy pronto, y por una serie de experiencias muy significativas como la enfermedad crónica de su hermano y la difícil situación de Alemania a partir de la Primera Guerra Mundial, que desembocó en la traumática experiencia nacionalsocialista y en la Segunda Guerra Mundial.

A pesar de todas las dificultades objetivas en las que se desarrollaron las primeras etapas de su larga vida, tuvo la oportunidad de una sólida formación en un Colegio distinguido, el del Santo Espíritu, y contó con un ambiente familiar propicio al estudio, pues no siempre se encuentra uno en su propia casa a los dieciocho años con un ejemplar de la Critica de la razón pura de Kant, ni con que su padre, prestigioso catedrático de Química farmacéutica de Marburgo, es además el rector de la Universidad en la que está cursando los estudios.

No obstante, la persistente influencia de su padre estuvo siempre dirigida hacia el estudio de las ciencias, hacia algo de lo que se pudiera obtener algún rendimiento, es decir, totalmente en contra de aquello para lo que Hans-Georg se sentía motivado. De ahí el también permanente conflicto entre las expectativas paternas y sus personales inclinaciones hacia la literatura, el arte, el mundo del espíritu y las humanidades.

Tal vez por eso las motivaciones más básicas en ese tiempo inicial de su formación le vinieron de la poesía de Stefan George y Rabindranaz Tagore o del pensamiento de Theodor Lessing y de Sören Kierkegaard. Y sus preocupaciones tenían relación primordialmente con la crisis cultural que se estaba viviendo, la crisis de la ciencia, que se iba convirtiendo cada vez más en peligroso dominio técnico y la creciente problematización del orgulloso proceso moderno. Como es bien conocido, en su momento tuvieron un gran impacto vital las experiencias de la catástrofe del Titanic y, por supuesto, de modo especial en Alemania las consecuencias de la derrota en Primera Guerra Mundial y las posteriores humillaciones.

\section{El encuentro con la filosofía}

Aunque sus primeros encuentros con la filosofía se produjeron en el ámbito del neokantismo de la Escuela de Breslau, la influencia más preponderante le ven-

1 Vid. J. Grondin, Hans-Georg Gadamer. Una biografia, Barcelona, Herder, 2000 y H.-G. GADAMER, Mis años de aprendizaje, Barcelona, Herder, 1996. 
dría de Martin Heidegger. En Breslau comenzó estudiando en 1918 una cierta interpretación neokantiana del Kant de la Crítica de la razón pura, de la que tal vez provenga su inicial animadversión hacia Kant, que luego tuvo que ser corregida a partir de la orientación aportada por los estudios de su amigo Gerhard Krüger. No obstante, todavía en Breslau a partir del semestre de 1918-19 tuvo otra experiencia dentro del neokantismo con las clases sobre "Teoría del conocimienton de Richard Hönigswald, a quien puede considerarse su primer maestro.

Pero, a pesar del posible atractivo de esta orientación neokantiana de Hönigswald, menos logicista y más abierta a los procesos vitales, Gadamer desde un comienzo se inclinó por una filosofía menos sistemática y más existencial y muy pronto sucumbió a los encantos del pensamiento heideggeriano, que se convirtió en su principal punto de referencia. Lo cual quedó patente ya desde sus primeros escritos, en los que se recoge la orientación que Heidegger estaba impartiendo en el semestre de verano de 1923 sobre una nueva Ontología en virtud de una Hermenéutica de la facticidad.

Por eso, cuando se trasladó a Marburgo, aunque siguió ligado principalmente a Nicolai Hartmann, el último eslabón de la neokantiana «Escuela de Marburgo" tras Cohen y Natorp, su alma filosófica quedó marcada por Heidegger para siempre. Y en ese cambio de Hartmann a Heidegger fue decisivo el semestre de verano de 1923.

Pues ese semestre Gadamer fue a Friburgo a estudiar a Aristóteles con Heidegger, aunque en realidad Hartmann se lo había encomendado a Richard Kroner, autor del libro De Kant a Hegel (1921), de quien lo consideraban en un principio discípulo en Friburgo. Pero el impacto que le produjo la "descarga eléctrica" de Heidegger le descubrió su propio camino filosófico. Y ya bajo esta influencia heideggeriana escribió sus primeros escritos; en especial cabe destacar la reseña crítica al libro de Hartmann Metafisica del conocimiento, en la que tras alabar el acercamiento de éste a la fenomenología, no aceptaba su recuperación de Aristóteles en términos de una ontología realista, sino que prefería la radicalización hermenéutica de la fenomenología, aprovechando otro modo de acceder a Aristóteles que estaba descubriendo a través de los seminarios con Heidegger en Friburgo (a quien, no obstante, todavía no citaba explícitamente).

${ }^{2}$ J. GRONDIN, Hans-Georg Gadamer, op. cit., pp. $110 \mathrm{s.}$ 
Así pues, en este momento crucial, Gadamer se situaba lejos del idealismo y del realismo. Por un lado, ya no podía proseguir el enfoque gnoseológico de las Escuelas neokantianas de Breslau y Marburgo, orientado por la «Teoría del conocimiento", ni tampoco su última derivación hacia la ontología realista de Hartmann, sino que se sintió atraído por la peculiar fenomenología de Heidegger, que empezó a desarrollarse en sentido hermenéutico y desde la que se anunciaba una nueva ontología: "Ontología (Hermenéutica de la facticidad)» era el título del curso de Heidegger en ese semestre de verano de 1923 en Friburgo.

\section{Filosofía y Filología}

La formación filosófica a Gadamer no le satisfizo del todo, a pesar del aprecio que le dispensaban sus mentores (Natorp, Hartmann, Kroner, Heidegger), tal vez porque se sentía inseguro, sobre todo tras el deslumbramiento y la fascinación experimentados ante la brillantez del lenguaje heideggeriano.

Al parecer, tanto esta falta de seguridad en sí mismo como el modo de entender - vivir- los conceptos filosóficos conforme a una experiencia más personal y cercana a su sentido poético y literario pudo impulsarle en 1925 a estudiar la carrera de filología clásica bajo la orientación de Paul Friedländer (quien por entonces estaba preparando su gran obra sobre Platón). También es posible que estuviera influido por la necesidad de acercarse al mundo de los griegos de un modo más riguroso, de un modo que pudiera cumplir de alguna manera las exigencias del pensamiento científico al que su padre le empujaba, aunque fuera por otro camino, en concreto el de una disciplina rigurosa dentro de las ciencias del espíritu. En cualquier caso, desde entonces, para Gadamer, el enfoque filosófico quedaba inseparablemente unido al filológico.

En este asunto recibió un impulso especial de otro pensador con el que estuvo muy vinculado: el teólogo Rudolf Bultmann, con quien también Heidegger mantuvo una estrecha amistad y una larga e intensa colaboración. Aunque con parsimonia, Gadamer confiesa haber aprendido mucho de la teología y del sentido humanista de Bultmann, sobre todo durante los muchos años que fue miembro de sus "Graeca» (reuniones semanales para leer durante horas los clásicos de la literatura griega).

Debido a este contacto con Heidegger y Bultmann, Gadamer se interesó por la teología, campo en el que había entrado seguramente a través de Kierkegaard 
y también de los estudios sobre historia de la hermenéutica de Dilthey. En este contexto acentuó la vinculación entre la filosofía y la filología, que ha marcado todo el posterior desarrollo de su hermenéutica, y asimismo incorporó el sentido humanista de la cultura a partir del estudio directo de los clásicos griegos. Le resultó un círculo muy acogedor en momentos en que precisamente se sentía inseguro e incluso rechazado por Heidegger, por lo que llegó a dudar de su capacidad e idoneidad para la filosofía. No obstante, el aprendizaje científico junto a Friedländer y el reconocimiento de su capacidad científica, refrendado por Heidegger (a quien hizo llamar el padre de Gadamer en 1928, cuando se encontraba moribundo en su lecho de muerte, para recomendarle la tutela de su hijo), le infundió nuevas fuerzas para proseguir su propio camino, apoyado en el campo filológico por Friedländer (y la complementaria ampliación bultmanniana) y en el filosófico por Heidegger, que se convertía desde entonces en su auténtico upadre espiritual»"

\section{El camino de la hermenéutica}

Con todo el bagaje filosófico y filológico antes aludido, con sus trabajos filosóficos y filológicos sobre el Protreptikós de Aristóteles, su interpretación del Filebo de Platón (base de su libro La ética dialéctica de Platón), Gadamer comenzó a forjar su peculiar talante hermenéutico.

La influencia principal de su propuesta provenía de Heidegger y de la tradición de la hermenéutica. Pero, aunque es indudable su heideggerianismo, ¿¿de qué Heidegger se trata?

Pues en el entretanto los acontecimientos políticos de la Alemania dominada por los nacionalsocialistas habían situado a Heidegger en una posición incomprensible - e incluso escandalosa - para muchos de sus discípulos. Y, aunque Gadamer dice no poder contar nada de lo que fuera testigo directo del tiempo que siguió al año 1933, de hecho no interrumpió del todo el contacto con Heidegger.

En 1936 fue a Fráncfort para escuchar una conferencia de Heidegger sobre «El origen de la obra de arte». A Gadamer le parece que "el Zaratustra nietzs-

${ }^{3}$ Ibíd., p. 176. 
cheano, maestro del eterno retorno [era] aquí el nuevo modelo» y de hecho Heidegger estaba entregado por entonces a la interpretación del pensamiento de Nietzsche, lo que luego recogió en su obra Nietzsche en dos volúmenes, según Gadamer, "verdadera contraparte a $E l$ ser y el tiempo». «No era Nietzsche», ni tampoco religión, sino "exploraciones» filosóficas, búsqueda de un lenguaje filosófico más allá de Hegel y de Nietzsche, capaz de rememorar (¿«repetir»?) el más antiguo comienzo del pensamiento griego, la búsqueda de posibilidades experienciales ${ }^{4}$

Sin embargo, la hermenéutica filosófica de Gadamer en Verdad y método, quien había estado a la sombra de Heidegger, con quien estudió en Friburgo primero y luego en Marburgo en los años veinte (la época de Ser y tiempo), adquiere un relieve propio al conectar su propuesta no directamente con el desarrollo de la Kehre ("vuelta" o "giro») heideggeriana, sino con el Heidegger de los primeros años veinte, con la "Hermenéutica de la facticidad».

Además de Heidegger, la hermenéutica de Gadamer incorpora las diversas tradiciones de la hermenéutica y las aportaciones de Schleiermacher y Dilthey, así como de la fenomenología del propio Husserl y la cultura humanística. En este sentido, no se prescinde de la hermenéutica como arte de la interpretación de los textos ni como doctrina del método de las filología en tanto que disciplina de las ciencias del espíritu. Que esta dimensión no sea la más profunda en la fundamentación de la hermenéutica no quiere decir que se prescinda de este nivel en la práctica hermenéutica.

El camino de la hermenéutica propiamente gadameriana no fue fácil ni rápido, como lo muestra el hecho mismo de que su intento de presentar los principios fundamentales de una hermenéutica filosófica cumplió el antiguo adagio horaciano, según el cual todo lo bueno necesita nueve años. Por fin, en 1959 reunió sus estudios sobre hermenéutica filosófica bajo el título de Verdad y método. Fue un largo proceso de elaboración de sus estudios sobre estética, filosofía de la hermenéutica y de la historia, tras los pasos de Dilthey, Husserl y Heidegger, con el fin de justificar los amplios ámbitos de la experiencia hermenéutica. Según el propio Gadamer, este intento hermenéutico evocaba la tradición de la

\footnotetext{
${ }^{4}$ Ibíd., p. 256.

${ }^{5}$ H.-G. GADAMER, Mis años de aprendizaje, op. cit., p. 213.
} 
metafísica occidental, pero rebasando el horizonte de la cultura dominante, a fin de recuperar sus fuerzas más originarias. Pero Gadamer dudaba si todavía era el momento oportuno o se había hecho tarde para hacer resonar una voz semejante en tiempos en los que predominaba una renovada conciencia de «emancipación» en el mundo intelectual.

\section{Hermeneutización ontológica histórica versus planteamiento epistemológico (Verdad antes que Método)}

En las últimas décadas del siglo XIX la filosofía se ocupó principalmente del fenómeno de la conciencia a través de un enfoque psicológico, que pretendía fundamentar la lógica y la teoría del conocimiento. Y, como los fenómenos de la conciencia se consideraban vivencias y su trabazón, vida, irrumpió asimismo un enfoque más radical que se configuró en forma de "filosofía de la vida".

"Tenemos hoy día que partir de la realidad de la vida" (decía Dilthey) y de sus formas de expresión (en virtud de un nuevo concepto de "espíritu objetivo", que abarca hasta el "espíritu absoluto" hegeliano). Porque la vida se autocomprende en la conciencia histórica. La filosofía vale como expresión de la vida, como su comprensión interpretadora, y el saber de sí mismo se efectúa en la conciencia histórica. Pero, entonces, la movilidad de la vida y la historia, ¿̨no excluyen el saber objetivo? ¿Dónde encontrar una base firme?

Si partimos "desde la vida", es en ella misma y en sus expresiones donde encontramos ya un cierto saber. El dato originario (Gegebenheit), para Dilthey, está en el nexo de vida y saber. El pensamiento surge de una necesidad vital y por eso habrá que preguntarse si se trata de un pensar inmanente a la vida o frente (¿contrario?) a la vida. Para Dilthey, la hermenéutica sería el medium de la conciencia histórica, para comprender la vida en sus expresiones.

Pero, según Gadamer, Dilthey se encontraba todavía escindido entre: 1) un planteamiento epistemológico, debido a su búsqueda de una fundamentación de las "ciencias del espíritu" (lo cual supone un resto de "cartesianismo epistemológico»), y 2) el análisis de la experiencia vital e histórica. De ahí que la siguiente

${ }^{6}$ Vid. M. HeidegGer, Ontologia. Hermenéutica de la facticidad (lecciones en el semestre de verano de 1923), Alianza, Madrid, 1999, pp. 93-94. 
aportación de la fenomenología consista en contribuir a la "superación del planteamiento epistemológicon?

De este clima arranca "el proyecto heideggeriano de una fenomenología hermenéutica». Según Gadamer, Heidegger está imbuido en sus comienzos por la tendencia común a Dilthey y Husserl ${ }^{8}$ a "concebir desde la vida», pero sin sus implicaciones epistemológicas.

Heidegger opone a la fenomenología eidética de Husserl una [fenomenología] "hermenéutica de la facticidad», en la que la facticidad del Dasein se erige en base ontológica del planteamiento fenomenológico. Lo cual implica una ontologización de la fenomenología.

Este nuevo enfoque constituye una crítica al idealismo, pero manteniendo la pretensión del planteamiento trascendental. Pues, según Gadamer, el proyecto heideggeriano (de Ser y tiempo) no escapa por completo al ámbito de la reflexión trascendental'.

La hermeneutización de la fenomenología puede mostrar el sentido de «toda una dimensión nueva", ganada en la interpretación trascendental de la comprensión por Heidegger. La estructura general de la comprensión alcanza su concreción en la comprensión histórica, en la que operan las vinculaciones de la costumbre, la tradición y las posibilidades de futuro. «El Dasein que se proyecta en su poder ser es ya siempre 'sido'n: factum existencial, facticidad (de la que brota toda posibilidad y comportarse libre).

"Tal es el quid de la hermenéutica de la facticidad" y de su oposición a la investigación trascendental de la constitución en la fenomenología de Husserl. He aquí una fórmula para expresar la unión o conexión entre transcendentalidad y facticidad histórica: transcendentalidad en la facticidad. El "presupuesto

${ }^{7}$ H.-G. GADAMER, Verdad y método, Sígueme, Salamanca, 1977, pp. 305 ss.

${ }^{8}$ También debe tenerse en cuenta la aportación del conde Yorck al concepto de "vidan: 1) vida es autoafirmación y la estructura de la vitalidad consiste en "analizan,, "dirimir" (Urteilung = enjuiciamiento; podría traducirse, a mi juicio, también como «discernimiento»); 2) correspondencia estructural de vida y autoconciencia (a partir de la Fenomenologia de Hegel: vivir implica asimilar, incorporar, apropiarse de lo extraño).

${ }^{9}$ H.-G. GADAMER, Verdad y método, op. cit., p. 320. 
irrebasable" es lo que al mismo tiempo «hace posible y limita todo proyectar»: la estructura existencial del Dasein.

El proyecto heideggeriano de fenomenología hermenéutica tiene el sentido de una "ontología fundamental", con la que Heidegger se proponía una renovación general del problema del ser (más allá de Dilthey y Husserl). Lo que el ser significa debe determinarse desde el horizonte del tiempo, pero con el plus de que "el ser mismo es tiempon, con lo cual se rompe todo el subjetivismo de la nueva filosofia [fenomenológica].

¿Hay entonces una fundamentación temporal de la validez objetiva? Según Gadamer, "la fenomenología de la conciencia del tiempo explica la fundamentación temporal de la validez objetiva», porque "Husserl no se quedó en la significación unívoca del ideal, de la que hablan las primeras Investigaciones lógicas, sino que intentó mostrar la identidad allí supuesta mediante su análisis del tiempo". A partir de lo cual Gadamer llega a afirmar lo siguiente: “Del concepto de síntesis pasiva y de la doctrina de las intencionalidades anónimas hay una línea que llega a la experiencia hermenéutica y que podría coincidir (...) con mi fórmula 'cuando se comprende, se comprende de un modo diferen$t e^{\prime}{ }^{10}$.

En virtud de este enfoque de ontología hermenéutica histórica, Gadamer ha aportado una reflexión sobre diversos aspectos de gran importancia para la filosofía contemporánea, por ejemplo: 1) la historicidad de la comprensión y, por tanto, el papel de la tradición, la autoridad y los prejuicios en la constitución de la realidad histórica, más allá de la subjetividad y la autorreflexión idealistas; 2) el lenguaje como experiencia constitutiva de la apertura al mundo; y 3) la universalidad de la experiencia hermenéutica y la nueva figura del problema de la verdad en relación con la historia.

\section{La hermenéutica en el debate filosófico contemporáneo}

La hermenéutica gadameriana constituye una alternativa frente a otras tendencias contemporáneas, como la filosofía analítico-lingüística, el «racionalismo

${ }^{1}$ H.-G. GADAMER, Verdad y método II, Sígueme, Salamanca, 1998, pp. 22 y 23. 
crítico" popperiano y el pragmatismo (expreso o camuflado). Pero incluso en el propio ámbito hermenéutico ha de hacer frente a diversas modalidades ${ }^{11}$.

El primer frente de debate con el que se encuentra confrontado el pensamiento hermenéutico gadameriano es el de Heidegger mismo. ¿En qué medida las «líneas fundamentales de una filosofía hermenéutica» como la de Gadamer es - como advirtió el propio Heidegger- "cosa de Gadamer», dado que el pensamiento heideggeriano ya había evolucionado hacia otras formas de pensar, ya fuera bajo la inspiración de Nietzsche o bien la de Hölderlin?

El segundo frente es el de la hermenéutica metodológica (Rothacker, Betti, Hirsch, Albert). La orientación diltheyana de la hermenéutica tenía el sentido de la búsqueda de una fundamentación específica de las "ciencias del espíritu", pero la innovación gadameriana - por influencia no sólo de Heidegger, sino según otros también de Bultmann- se sitúa, más allá del metodologismo y epistemologismo reinantes, en el ámbito más radical de la experiencia ${ }^{12}$.

En tercer lugar, Gadamer tuvo que defenderse de los ataques procedentes de una hermenéutica que incorporaba la "crítica de la ideología" y se abría a las emergentes ciencias sociales. Además de proporcionarle una mayor resonancia social por su dimensión política, esta polémica (principalmente con Habermas y Apel) ha sido, a mi juicio, la más fecunda para la ampliación y transformación del pensamiento hermenéutico contemporáneo ${ }^{13}$.

Y, finalmente, en sus últimos años Gadamer respondió con renovada vitalidad a las críticas (explícitas e implícitas) provenientes de la denominada «deconstrucción", impulsada por J. Derrida, que representa una amalgama de fenome-

11 En ocasiones entremezclándose por diversas vías históricas: vid., por ejemplo, G. VATTIMO, El fin de la modernidad, Barcelona, Gedisa, 1986; Las aventuras de la diferencia, Barcelona, 1986; T. OÑATE, El retorno griego de lo divino en la postmodernidad, Madrid, Alderabán, 2000; M. Á. QUINTANA PAZ, Normatividad, interpretación y praxis: Wittgenstein en un giro bermenéutico-nibilista, Salamanca, Publicaciones de la Universidad Pontificia de Salamanca, 2005.

12 Vid. J. Conill, El enigma del animal fantástico, Tecnos, Madrid, 1991; El poder de la mentira. Nietzsche y la politica de la transvaloración, Tecnos, Madrid, 1997.

13 Vid. R. BUBNer, K. Cramer y R. WIEHL (eds.): Hermeneutik und Dialektik, Tubinga, Mohr, 1970; J. HABERMAS, D. HENRICH y J. TAUBES (eds.): Hermeneutik und Ideologiekritik, Fráncfort, Suhrkamp, 1971; J. CoNiLL, El crepuisculo de la metafisica, Barcelona, Anthropos, 1988. 
nología heideggeriana, algo de Nietzsche y del estructuralismo, amén de cierta mística judía, desde la que pretende poner en cuestión una vez más la metafísica, la tradición humanista e incluso el horizonte hermenéutico del sentido.

\section{¿Más allá de la hermenéutica?}

El complejo proyecto heideggeriano de una «fenomenología hermenéutica» estaba en un comienzo imbuido por la tendencia (típica de la época) de pensar o "concebir desde la vida". Pero su ontologización (por afán de superar el planteamiento epistemológico) dejó en suspenso una serie de cuestiones que el camino gadameriano, a mi juicio, tampoco ha resuelto ${ }^{14}$.

El análisis gadameriano de la historicidad de la comprensión pone acertadamente de relieve diversos aspectos del ser histórico, donde la distancia temporal es productiva hermenéuticamente, como los prejuicios, la tradición, el poder de la historia en la conciencia de la historia efectual, la fusión de horizontes, la anámnesis, el círculo hermenéutico, la anticipación, etc. Pero se detectan insuficiencias como las siguientes: 1. A pesar de que su análisis de la estructura de la conciencia de la historia efectual remite a la de la experiencia y se reconoce que ésta experimenta realidad y es ella misma real, el pensamiento hermenéutico de Gadamer se resiste a considerar la historicidad desde la realidad ${ }^{15}$. 2. Por otra parte, aunque caracteriza a esta conciencia de la historia efectual por su "apertura a la experiencia» (Erfahrungsbereitschaft), entiende e interpreta esta apertura primordialmente mediante la estructura lógica de la pregunta ${ }^{16} .3$. Además, deja sin resolver la aporía heideggeriana de la relación entre naturaleza e historia, entre vida natural e histórica.

Estas insuficiencias quedan mejor resueltas, a mi juicio, en la línea de la filosofía española que prosigue y enriquece, por ejemplo, Laín con su "analítica de

14 Hans-Georg GaDamer, Verdad y método, op. cit., p. 329. Vid. el abismo entre el hombre y el animal establecida por Martin Heidegger en Carta sobre el humanismo.

15 Sobre las insuficiencias del análisis gadameriano de la experiencia, vid. Jesús CoNILL, $E l$ enigma del animal fantástico, y "Concepciones de la experiencia", Diálogo filosofico, n. 41 (1998), pp. $148-170$.

${ }^{16}$ Histórica y experiencial, sí, pero, al reducirse al esquema aportado por la lógica de la pregunta, deja fuera el hecho de la expectación y de la "espera", que sin embargo ha sido atendido por todo el mejor pensamiento español (Unamuno, Machado, Ortega, Zubiri, Laín Entralgo y también Julián Marías). 
la esperanza» y su concepción estructurista-dinamicista del cuerpo humano ${ }^{17}$. Laín propuso tempranamente una nueva "analítica de la existencia», diferente de la heideggeriana de Ser y tiempo y cuyo punto de partida no fuera ya el modo de ser de la pregunta, sino un modo de ser más radical. Laín vislumbró que podría hablarse del "temple de la espera", al cual pertenecerían como formas derivadas la esperanza y la desesperanza, y lograr un análisis de este fenómeno de la espera, tomado como orientación originaria. A lo que debe añadirse otra característica peculiar consistente en que este estudio de Laín afronta el problema de la realidad biológica de la espera, destacando "el decisivo papel del cuerpo en la actividad de esperar ${ }^{18}$.

La Analítica hermenéutica podría quedar superada por la noológica (o al menos completada mediante el descubrimiento de estratos más profundos de la experiencia vital e histórica) y la ontología de la historicidad (en versión de la conciencia efectual) quedaría superada por una metafisica de la realidad histórica (aportando nociones como historicidad real, posibilidades reales, cuasi-creación y capacitación $)^{19}$.

Para seguir esta nueva vía alternativa (propia de la filosofía hispana), tras los procesos de hermeneutización de la fenomenología, hay que desontologizar (o al menos abstenerse de ontologizar tal hermeneutización) y quedarse con la valiosa aportación "analítica» de la facticidad, incluso de la transcendentalidad en la facticidad. Con lo cual el impulso filosófico hacia lo originario nos conduce a una pugna de analíticas, en la que no hay por qué desdeñar la nueva «insistencia analítica» que ofrece la transformación de la fenomenología que se produjo en los ya pensadores clásicos de la filosofía española contemporánea (Ortega, Zubiri, Laín) ${ }^{20}$.

17 Pedro LaÍn, La espera y la esperanza, Madrid, Alianza, 1984 (2. ${ }^{a}$ ed.); vid. J. ConiLL, «Laín Entralgo y Zubiri. De la analítica de la existencia a una concepción estructurista-dinamicista del cuerpo humanom, Pensamiento, n. 221 (2002), pp. 177-192.

18 Pedro Laln, Descargo de conciencia, Madrid, Alianza, 1989, p. 493.

19 Vid., a título de ejemplo, J. ORTEGA Y GASSET, Unas lecciones de metafisica, Madrid, Alianza, 1968; X. ZUBIRI, Estructura dinámica de la realidad, Madrid, Alianza, 1989; J. Marfas, Antropologia metafisica, Madrid, Revista de Occidente, 1973.

${ }^{20} \mathrm{~J}$. CONILL, «La transformación de la fenomenología en Ortega y Zubiri. La postmodernidad metafísican, en J. San Martín (ed.), Ortega y la fenomenologia, Madrid, UNED, 1992, pp. 297 312. 
La visión de la vida humana como realidad radical (en Ortega) y el descubrimiento de que todo acto humano (intelectivo, volitivo o afectivo) tiene su comienzo en la impresión primordial de realidad (según Zubiri) constituyen hitos de la superación metafisica de la fenomenología en el sentido de Husserl y se sitúan también más allá de la ontología fundamental (del sentido del ser) en Heidegger, porque están referidas y apoyadas en una nueva noción de realidad, de manera que no son simplemente "Ontología», sino que son formalmente "Metafísica»" ${ }^{21}$.

A través de esta alternativa, la hermeneutización de la filosofía contemporánea no tiene por qué aceptar que el camino de la experiencia conduce irremediablemente al nihilismo y al pragmatismo. Antes bien, una tarea de la filosofía actual consiste en aceptar el primado de la experiencia, pero sin caer en sus posibles consecuencias nihilistas y ciegamente pragmáticas.

Es más, un mejor análisis de la experiencia —impulsado incluso por la genealogía - puede contribuir a configurar una crítica de la razón impura, que prosiga la crítica de la razón - como tarea permanente de la filosofía-, arrancando del espesor de la experiencia vital y sin regirse por la lógica o por la metodología. Porque no nos encontramos ya siempre sólo, ni prioritariamente, en la razón reflexivo-formal, o en diálogo o en la argumentación, sino que estamos ya siempre y primordialmente en la experiencia histórica.

Así que, si hay que hacer frente a la autodestrucción nihilista y pragmatistacontextualista de la razón, es decir, a la denominada "crítica total de la razón" ${ }^{22}$, a la vez hay que ser conscientes del riesgo que se corre entonces de supeditarse de nuevo a la coerción epistemológica del metodologismo moderno y recaer en un "cartesianismo epistemológico" ${ }^{23}$. Ahora bien, entre ambos peligros hay una tercera posibilidad, la que media lógos y experiencia en el concepto de una "razón

21 Y esto dicho en tiempos que he calificado de criptometafisica (más que de la - hasta la saciedad reiterada- "época posmetafísica"). Vid. Jesús CONILL, El crepuisculo de la metafisica, op. cit., y "Filosofia sin criptometafísica», en Metafisicos actuales, Fundación Fernando Rielo, Madrid, 2003, pp.67-89.

${ }^{22}$ K.-O. APEL, «Die Herausforderung der totalen Vernunftkritik und das Programm einer philosophischen Theorie der Rationalitätstypen", Concordia, 11 (1987), 2-23.

${ }^{23}$ H.-G. GADAMER, Verdad y método, op. cit. 
experiencial, ${ }^{24}$. Es ésta una fórmula que, contando indudablemente con precedentes clásicos antiguos y modernos, prosiguen ciertas filosofías contemporáneas y de modo especial la tradición de la filosofía española (desde el usentimiento trágico" de Unamuno hasta la "razón vital» de Ortega, la "razón poética» de Zambrano y la "razón sentiente" de Zubiri, con sus valiosas prolongaciones antropológicas y metafísicas en Pedro Laín y Julián Marías).

${ }^{24}$ Vid. J. CONILL, El enigma del animal fantástico, parte II: «Hermenéutica crítica de la razón experiencial"; "La verdad de la razón experiencial», en J. A. NICOLAS y M. J. FRÁPOLLI (eds.), Verdad y experiencia, Granada, Comares, 1998, pp. 181-197; El poder de la mentira. 